\section{Rubber hands 'feel' touch that eyes see}

Illusions have historically been of great use to psychology for what they can reveal about perceptual processes. We report here an illusion in which tactile sensations are referred to an alien limb. The effect reveals a three-way interaction between vision, touch and proprioception, and may supply evidence concerning the basis of bodily selfidentification.

Each of ten subjects was seated with their left arm resting upon a small table. A standing screen was positioned beside the arm to hide it from the subject's view and a life-sized rubber model of a left hand and arm was placed on the table directly in front of the subject. The subject sat with eyes fixed on the artificial hand while we used two small paintbrushes to stroke the rubber hand and the subject's hidden hand, synchronising the timing of the brushing as closely as possible.

After ten minutes, subjects completed a two-part questionnaire that requested an open-ended description of their experience and asked them to affirm or deny the occurrence of nine specific perceptual effects (Fig. 1). The completed questionnaires indicated that subjects experienced an illusion in which they seemed to feel the touch not of the hidden brush but that of the viewed brush, as if the rubber hand had

We hypothesized that this illusion involves a constraint-satisfaction process operating between vision, touch and proprioception - a process structured by the correlations normally holding among these modalities. Specifically, a connectionsensed the touch.

ist model suggested that the illusion's spurious reconciliation of visual and tactile inputs relies upon a distortion of position sense.

This was tested in a second experiment by exposing subjects to the conditions of our first experiment for a prolonged period, and then probing for signs of a distortion of proprioceptive information. Both before and after the viewing period, subjects completed a series of three intermanual reaches. With eyes closed, the right index finger was drawn along a straight edge below the table until it was judged to be in alignment with the index finger of the left hand, which rested on the table in the same position as during the exposure period. Subjects' reaches after experiencing the illusion were displaced rightward toward the rubber hand, the magnitude of this displacement varying significantly in proportion to the reported duration of the illusion (Fig. 2).

A control group in which a small asynchrony was introduced between the brushing of the two hands reported low prevalence of the illusion (mean $7 \%$ of the exposure period compared to $42 \%$ for the synchronously brushed group; $P<0.01$ ), and failed to display reach displacement in the direction of the artificial hand (mean displacement $13 \mathrm{~mm}$ away from the rubber hand, compared with $23 \mathrm{~mm}$ towards it in the experimental group; $P<.04)$.

This illusion belongs to a class of perceptual effects involving intersensory bias ${ }^{1-4}$. In closely related work, Ramachandran et al. got phantom limb patients to view their intact arm in a mirror, so that their amputated arm appeared to have been resurrected. Several subjects viewing the reflection as the intact arm was touched reported feeling the touch in the amputated (phantom) limb ${ }^{5}$.

Also relevant is the finding of cells in the

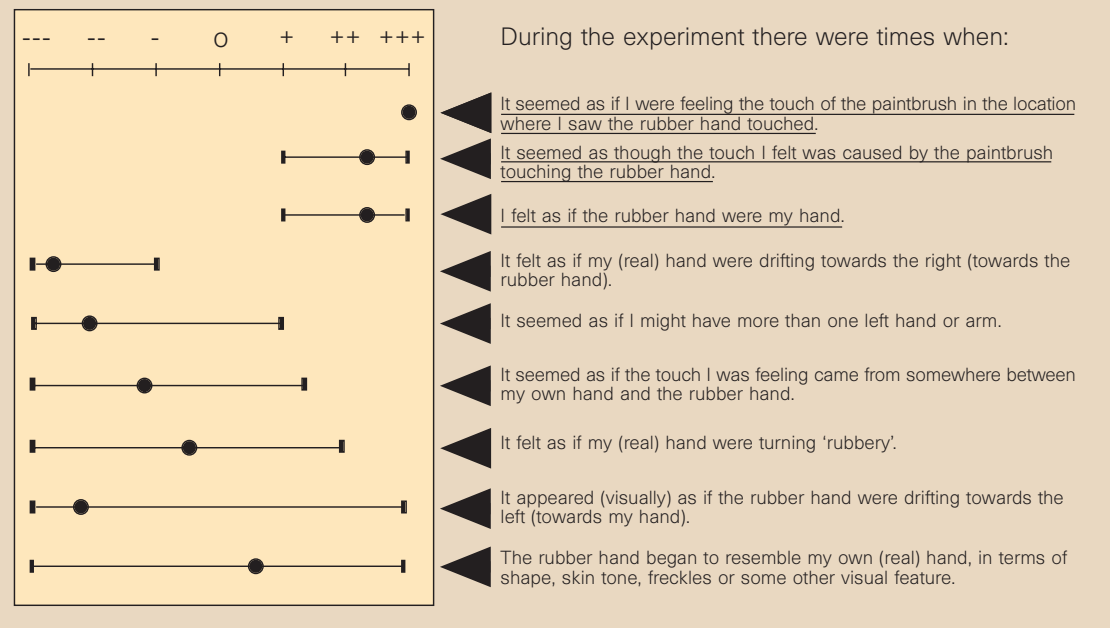

Figure 1 Questionnaire results. The questionnaire included the nine statements shown, presented in a random order. Statements describing the predicted phenomena are underlined. Subjects indicated their response on a seven-step visual-analogue scale ranging from 'agree strongly' (+++) to 'disagree strongly' $(---)$. Points indicate mean responses. Bars indicate response range. The questions underlined showed a statistically significant tendency to evoke affirmative responses $(P<0.002$ for underlined questions, $P<0.018$ after correcting for multiple comparisons).

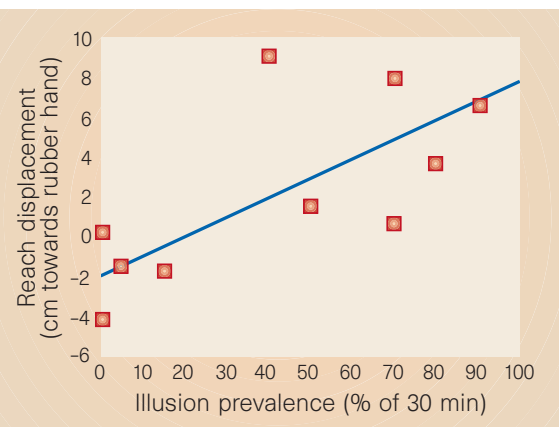

Figure 2 Results of reaching experiment. The $x$-axis indicates the percentage of the 30-min viewing period during which the ownership illusion was experienced. The $y$-axis indicates displacement of the three reaches made after the viewing period from the three made before, calculated as the difference between the means in the two groups. The data is fitted with a least-squares regression line ( $y$ $\left.=0.09 x-1.4, r^{2} 0.47, p<0.03\right)$.

premotor cortex of monkeys which respond both to tactile stimulation of a particular body region and to visual perception of an object approaching that area ${ }^{6}$. The connectionist network referred to above features a layer of units with analogous response properties, units that appear to be necessary for the relevant cross-modal interactions to occur.

It has been proposed that the body is distinguished from other objects as belonging to the self by its participation in specific forms of intermodal perceptual correlation $^{7,8}$. Subjects in our first experiment who referred their tactile sensations to the rubber hand also consistently reported, in both sections of the questionnaire, experiencing the rubber hand as belonging to themselves. Indeed, eight of ten subjects spontaneously employed terms of ownership in their free-report descriptions, for example: "I found myself looking at the dummy hand thinking it was actually my own."

While the rubber hand illusion does not tell us precisely what ingredient might make only certain forms of intermodal correlation relevant to the self, it does show that intermodal matching can be sufficient for self-attribution.

Matthew Botvinick, Jonathan Cohen Department of Psychiatry, University of Pittsburgh, and Department of Psychology, Carnegie Mellon University, Pittsburgh, Pennsylvania 15213, USA email:mmb@crab.psy.cmu.edu

1. Hay, J.C. \& Pick, H.L. \& Ikeda, K. Psychon. Sci. 2, 215-216 (1965).

2. Tastevin, J. L'Encephale 1, 57-84 (1937).

3. DiZio, P, Lathan, C.E. \& Lackner, J.R. Journal of Neurophysiology. 70, 1578-1584 (1993).

4. Witkin, H.A, Wapner, S. \& Leventhal, T. J. Exp. Psychol. 43, 58-67 (1952).

5. Ramachandran, V.S., Rogers-Ramachandran, D. \& Cobb, S. Nature 377, 489-490 (1995).

6. Graziano, M. \& Gross, C. Science 266, 1054-1057 (1994).

7. Lewis, M. \& Brooks-Gunn, J. Social Cognition and the Acquisition of the Self(Plenum, New York, 1979).

8. Bahrick, L.E. \& Watson, J.S. Developmental Psychology 21, 963-973 (1985) 